\title{
Atenção primária à saúde e territórios latino-americanos marcados pela violência
}

\author{
Ana Elisa Medeiros Barbar ${ }^{1}$
}

Como citar Barbar AEM. Atenção primária à saúde e territórios latino-americanos marcados pela violência. Rev Panam Salud Publica. 2018;42:e142. https://doi.org/10.26633/RPSP.2018.142

\begin{abstract}
RESUMO
O artigo busca discutir singularidades do trabalho da atenção primária à saúde (APS) em contextos onde a violência, apoiada fortemente pelo uso de armas de fogo, tem expressão rotineira, crônica e sistêmica, e dimensionar a potência da APS enquanto canal de empoderamento da comunidade onde a violência armada é uma vulnerabilidade expressiva. Com base na Declaração de Alma-Ata e nos princípios da APS enumerados por Barbara Starfield, e com enfoque na participação comunitária e na perspectiva de determinantes sociais de saúde, a discussão tem como ponto de partida realidades observadas em países da América Latina e os compromissos voltados ao acesso e cobertura universal à saúde previstos no terceiro dos Objetivos de Desenvolvimento Sustentável. É necessário qualificar a caracterização qualitativa e quantitativa da violência armada e de suas consequências, a partir de uma abordagem intersetorial com ampla participação comunitária na busca por respostas coerentes e significativas. Também é preciso garantir a proteção e a segurança dos profissionais para que a presença dos serviços de saúde nos espaços seja contínua.
\end{abstract}

Palavras-chave Atenção primária à saúde; violência; participação social; acesso aos serviços de saúde.

A América Latina é um território geográfico e cultural extremamente diverso. Entretanto, seus países são reconhecidos por terem em comum uma acentuada desigualdade social e altos níveis de concentração de renda por um grupo extremamente pequeno de pessoas, além de políticas públicas em geral insuficientes ou de resultados intermitentes, que dificultam a transformação dessa realidade (1). A condição de pobreza e escassez de recursos que atinge grande parte da população produz e reproduz graves processos de exclusão social, tais como dificuldades de acesso a condições dignas de moradia, saneamento, água e energia

\footnotetext{
Comitê Internacional da Cruz Vermelha, Brasília (DF),
} Brasil. Correspondência: aembarbar@gmail.com elétrica, segurança alimentar com variedade e qualidade nutricional e serviços de saúde e educação $(2,3)$.

Com crescimento cada vez mais acelerado, as megacidades são reconhecidas como espaços altamente representativos dos problemas mencionados. Porém, o meio rural, ainda que com particularidades, não está excluído da dinâmica da desigualdade, nem está isento de suas consequências (4).

Além das fragilidades por vulnerabilidades sociais expressivas, a presença de grupos armados cuja prática econômica é baseada em atividades ilegais representa muitas vezes, para moradores de bairros e regiões inteiras, um convívio diário com a violência armada. Tais grupos recebem diferentes nomes, como "gangues", "guerrilhas", "facções", "maras" ou "milícias", de acordo com suas características. Nesses contextos, a violência é exercida ativa e reativamente como forma de controle de corpos, de espaços e de práticas, e os atos violentos são cometidos tanto por membros dos grupos quanto por atores do Estado, já que estes também fazem parte desse complexo cenário (4). A ampla circulação de armas no território favorece sua utilização como meio de intimidação, defesa ou agressão direta, aumentando a exposição da população ao risco de ferimentos e mortes, intencionais ou acidentais (2). A violência ainda é responsável por deslocamentos forçados, por separações de familiares e restrições de mobilidade a moradores das áreas 
afetadas $(3,5)$. Nesses contextos, a redução ou impedimento de acesso a serviços de saúde e educação se dá diretamente, como por bloqueio de vias ou falta de segurança para deslocamento até os serviços $(3,6,7)$, mas também indiretamente, pela dificuldade de alocação de profissionais nessas áreas ou por redução de horários de funcionamento (8).

Tal realidade pode ser observada, em diferentes intensidades, em regiões do Brasil, Colômbia, Peru, México, El Salvador e Honduras, entre outros países. Muitas vezes, a apresentação dessa realidade pelos veículos de comunicação é vinculada a respostas reducionistas, promovendo o aumento de violações aos direitos humanos (9).

Em relação à descrição geral do contexto em questão, não há consenso quanto à nomenclatura: alguns autores se referem ao cenário como "violência não-convencional" para descrever o "fenômeno da violência organizada e não-organizada em mãos de diferentes atores, estatais e não-estatais, que dá lugar a consequências humanitárias similares às de um conflito armado" $(3$, p. 6 , tradução da autora). Essa definição vem sendo utilizada por agências das Nações Unidas, assim como por algumas organizações não governamentais, para discutir a temática sob diferentes ângulos, embora ainda não seja utilizada em debates voltados à saúde. O Comitê Internacional da Cruz Vermelha utiliza em sua atuação o termo "outras situações de violência" para se referir a cenários semelhantes, com circunstâncias bem definidas para sua aplicação (3). Outros termos utilizados para descrever o contexto na academia - e fora dela - são os mais genéricos "violência armada" e "violência urbana", que serão então adotados ao longo deste texto para fazer referência a contextos onde a violência é exercida fortemente pelo emprego disseminado de armas de fogo, com expressão rotineira, crônica e sistêmica, e consequências significativas para a qualidade de vida, mobilidade e integridade física e mental de moradores destes contextos.

$\mathrm{O}$ presente artigo busca discutir singularidades do trabalho da atenção primária à saúde (APS) em contextos de violência armada e dimensionar a potência da APS enquanto canal de empoderamento da comunidade onde tal violência é uma vulnerabilidade expressiva. Com base na declaração de Alma-Ata (10) e nos princípios da APS enumerados por
Barbara Starfield (11), e com enfoque na participação comunitária e na perspectiva de determinantes sociais de saúde, a discussão tem como ponto de partida as realidades observadas em diversos países da América Latina e os compromissos previstos no terceiro Objetivo de Desenvolvimento Sustentável, nomeadamente "assegurar uma vida saudável e promover o bem-estar para todos, em todas as idades" (12). Cabe destacar que este texto não irá se aprofundar nas significativas e transversais questões de gênero, embora sejam uma dimensão marcante do fenômeno da violência (13).

\section{SINGULARIDADES DA APS EM CONTEXTO DE VIOLÊNCIA ARMADA}

O trabalho da APS presume um olhar integral, longitudinal - cuidado focado na pessoa, e não na doença, ao longo do tempo (14) - e participativo para o contexto em que as práticas de cuidado se inserem. Assim, é coerente que leve em consideração as singularidades do território, da população, da história e das relações que se estabelecem em cada espaço de atuação para a construção de narrativas significativas de cuidado a partir de todas as vozes e saberes presentes $(14,15)$.

Como um dos elementos que atravessa as práticas da APS, o fenômeno da violência é multidimensional, com causas e determinantes complexos, e sua compreensão não é tarefa fácil: a violência é parte dos determinantes sociais da saúde e dos elementos condicionantes ao acesso da população a espaços de cuidado, exigindo reflexão ampliada a respeito da realidade das comunidades afetadas, com a atenção de não extrair das mesmas seu potencial de vida e resiliência. Mais desafiador ainda é ofertar cuidado à saúde nos territórios marcados pela violência armada, onde relações pessoais, comunitárias e institucionais estão permeadas por suas consequências.

De acordo com a Organização Mundial da Saúde (OMS), define-se o conceito de violência como "uso intencional de força física ou poder, em ameaça ou na prática, contra si próprio, outra pessoa ou contra um grupo ou comunidade, que resulta ou possa resultar em sofrimento, morte, dano psicológico, desenvolvimento prejudicado ou privação" (2, p. 1165). Em relação à tipologia da violência, também a OMS explicita as categorias "violência interpessoal", "violência autoinfligida", e "violência coletiva", esta última praticada por grupos organizados (dentre eles Estados nacionais) para alcançar objetivos políticos, econômicos ou sociais.

Há um entrave em identificar o problema da violência enquanto fenômeno sistêmico a partir de categorizações e práticas que privilegiam o âmbito individual de cada evento de violência, o que dificulta a busca por respostas mais pertinentes frente à realidade observada. A alternativa "violência coletiva" não aparece empregada para o cenário de violência armada referido neste artigo: a ausência de legitimação jurídico-política dos grupos perpetradores da violência, por exemplos gangues ou facções, impede o reconhecimento dos mesmos de um ponto de vista de sua "organização". Também as implicações sociais e ideológicas da constatação oficial de atos sistemáticos de violência por determinados atores, como milícias, fazem com que tais eventos sejam preteridos por esferas competentes.

Da mesma forma, a maneira como a realidade é apreendida por indicadores de saúde traz dificuldades para a superação da dimensão individual de expressão da violência. A partir do olhar ampliado das políticas de saúde, a caracterização e a tipologia de violência interpessoal ou autoinfligida, propostas pela OMS, são frequentemente utilizadas, apoiadas por indicadores epidemiológicos tradicionais, como taxas de homicídios e incidência de lesões por armas de fogo. O uso desses marcadores não favorece a distinção entre atos de violência interpessoal isolados e violência enquanto prática crônica de controle, aplicação violenta da lei ou disputa de poder. A complexidade da coleta de dados para documentar e tipificar uma série de eventos de violência física, ameaças e homicídios, faz com que a questão seja abordada de forma fragmentada, sob esforços temáticos específicos, como é o caso da violência de gênero e da violência sexual. Dados sobre eventos considerados de ordem "criminal", coletadas por órgãos de segurança pública, ou ainda toda a discussão relativa à circulação de armas, por exemplo, não são debatidos amplamente com interlocutores de saúde. Assim, a necessidade de formulação do problema enquanto elemento de política pública, cuja resposta também inclui o fortalecimento da APS assim como previsto na Declaração de 
Alma-Ata, fica comprometido quando há adoção de políticas segmentadas e unidepartamentais.

Sobre a questão da inclusão de dados sobre violência, sua tipologia e caracterização, em políticas públicas de saúde, o artigo de Dahlberg e Krug (2) é leitura obrigatória e faz uma excelente reflexão, ainda que não enfoque os recursos específicos da APS no processo de análise do problema e formulação de respostas. Também o artigo de Minayo (16), já em 1994, apontava a dificuldade de descrever qualitativa e quantitativamente o cenário brasileiro de violência.

Além da complexidade de apreensão do contexto de violência armada, o desafio de extrapolar o espaço microdimensional da clínicano atendimento assistencial a vítimas de violência faz com que cada evento capturado pelos profissionais de saúde tenha um ciclo breve, com início no acolhimento da queixa e encerramento no processo de notificação do evento violento, seja por registros em prontuários ou envio das informações pertinentes a setores de gestão de dados. Observa-se que análises situacionais e consequentes respostas estruturais e ampliadas para a dinâmica de violência requerem um esforço de interpretação local da informação produzida nesses atendimentos individuais. Ademais, além da produção de informação, o processo burocratizado frente a um evento de violência desaloja a perspectiva humana do cuidado em saúde e dificulta a coordenação do cuidado desde um ponto de vista centrado na pessoa, tão caro à APS (17).

As discussões em espaços grupais e comunitários é atividade significativa para compreender o impacto da violência nos territórios, ainda que seja necessário um alto grau de confiança entre população e profissionais de saúde, para que as experiências e impressões possam ser compartilhadas (7). Não há dúvidas de que a APS é o nível de cuidado privilegiado para a participação comunitária no desenho e implementação de atividades em saúde. Parece, então, de extrema importância que a voz da população seja horizontal na identificação de demandas e no entendimento da realidade dentro da qual a APS vai se desenvolver (15). Assim, o engajamento da comunidade nos espaços e práticas pode ser elementochave para que o cotidiano de violência seja abertamente considerado, caracterizado e integrado ao cuidado em saúde, e para que intervenções da APS em cada território sejam não apenas contextualizadas, mas transformadoras (18).

Justamente para tal dimensão transformadora, é necessário apontar que a presença contínua no território, a longitudinalidade do cuidado e a construção de vínculos, assim como seu papel acolhedor como porta de entrada ao sistema de saúde, conferem à APS o grande potencial de oferecer um caminho sustentável de atenção à saúde em todos os contextos, mas especialmente naqueles mais fragilizados. Desde seguranças macropolíticas de acesso equitativo até a segurança tão pessoal e humana de reconhecer faces familiares no processo de cuidado, a APS é um caminho de cidadania e empoderamento para populações cronicamente afetadas por exclusão social e privação de direitos.

Além dos desafios para compreensão do fenômeno da violência armada e integração do cenário em suas práticas, os profissionais enfrentam desafios pessoais para a atuação nesses territórios: não se pode evitar por completo a exposição a riscos relacionados à violência, $\mathrm{e}$ a proteção dos trabalhadores de saúde enquanto prestadores imparciais de cuidado é por vezes desrespeitada pelos atores envolvidos, estatais ou não. Uma observação pertinente para a preservação do lugar de imparcialidade é a importância de manutenção do sigilo para as informações produzidas em espaços de APS, cujas garantias legais são, em algumas circunstâncias, frágeis ou simplesmente desrespeitadas.

Machado e Daher (8), assim como Polaro et al. (7) discutem, entre outras questões, o impacto da violência urbana sobre o trabalho e o bem-estar dos profissionais de APS. A vivência de situações psicologicamente mobilizadoras, ataques, ameaças e coações durante o trabalho é uma infeliz realidade que deve ser monitorada e tratada adequadamente.

Para que o acesso da população ao cuidado em saúde seja concretizado, é necessário garantir que os serviços também sejam resilientes ao contexto, para além de práticas e intervenções pertinentes. Os espaços devem considerar a segurança dos profissionais e, ao mesmo tempo, evitar barreiras entre a população e as equipes de saúde, assim como os horários de trabalho devem equilibrar os riscos à segurança dos profissionais e a necessidade de atenção da população. Uma construção de longo prazo desse equilíbrio, com ampla mobilização social e apoio da governança responsável, para encontrar respostas válidas para todos é necessária para que a APS possa se desenvolver de forma eficiente $e$, de fato, ampliar o acesso da população ao cuidado $(3,5)$.

Um último (e talvez maior) desafio para o trabalho da APS frente à violência armada é responder às demandas de cuidado que surgem da comunidade e das pessoas, já que muitas das consequências da violência se expressam clinicamente de forma mais sutil, por queixas de pressão arterial aumentada ou crises de pânico, por exemplo. Deve-se ainda pensar nas vítimas secundárias da violência, que não sofreram o evento, mas foram impactadas por sua rede social direta, como os familiares de pessoas assassinadas ou desaparecidas. Também aqui a compreensão ampla do contexto, o monitoramento compartilhado de eventos adversos (como mortes, sequestros, tiroteios), a escuta cuidadosa e atenta na clínica e o trabalho multidisciplinar podem ajudar a responder de forma mais efetiva, aliando-se à resposta biomédica. O caráter multidisciplinar da APS, previsto em Alma-Ata, assim como sua proximidade à realidade vivida por cada pessoa cuidada, são recursos importantes para que a dimensão de violência seja integrada de forma não limitante ao projeto de cuidado individual e à resposta coletiva local que correspondem ao planejamento de trabalho de cada serviço de saúde, em todas as dimensões de cuidado.

\section{CONCLUSÃO}

A APS não pretende ser nem deve ser entendida como solução universal para as questões que afligem a sociedade. A adoção de políticas intersetoriais, estabelecimento de sistemas de saúde robustos e efetivos, além do comprometimento político com a equidade de acesso e a participação popular na formulação de respostas às suas próprias demandas são requisitos sine qua non para que nenhuma intervenção carregue, sozinha, o peso da transformação de realidades tão desiguais e excludentes, como observadas em grande parte do continente americano.

Apesar disso, deve-se reconhecer que, por seu caráter integral, multidisciplinar, participativo e longitudinal, a APS é um caminho extremamente potente para implementar processos de coordenação, cidadania e cuidado. Seu papel no 
reconhecimento e validação da narrativa comunitária sobre a realidade de violência em que se insere é único e deve ser valorizado - tanto no aspecto objetivo de caracterização da realidade, quanto nas experiências individuais da clínica. Por fim, devem-se explorar na APS as possibilidades de construção horizontal, junto à comunidade, de respostas sustentáveis garantidas pela continuidade do cuidado e da presença das equipes de APS em cada contexto afetado pela violência armada.
O espaço limitado deste artigo não esgota os questionamentos que qualificam o trabalho da APS em contextos de violência armada, abordando o cenário com visão ampliada e intersetorialidade de ações, ampliando o acesso da população a serviços de saúde e garantindo a participação comunitária na transformação de suas realidades sem abandonar a proteção e a segurança dos profissionais de saúde. Porém, espera-se que os pontos discutidos possam contribuir para destacar a

\section{REFERÊNCIAS}

1. Andrade LOM, Pellegrini Filho A, Solar O, Rígoli F, Salazar LM, Serrate PCF, et al. Social determinants of health, universal health coverage, and sustainable development: case studies from Latin American countries. Lancet. 2015;385:1343-51.

2. Dahlberg LL, Krug, EG. Violência: um problema global de saúde pública. Cienc Saude Coletiva. 2007;11(Sup):1163-78.

3. Stein S, Walch C. Implicaciones humanitarias de la violencia no convencional en el Triángulo Norte de Centro America y Mexico. Social Science Research Council/ Conflict Prevention and Peace Forum: Nova Iorque; 2016. Disponível em: https:/ /www. ssrc.org / publications / view / 451841138F7B-E711-80C6-005056AB0BD9/ Acessado em agosto de 2018.

4. Minayo MCS. Violência e educação: impacto e tendências. Rev Pedag. 2013;15(31): 249-64.

5. Cue W, Nuñes-Flores VR. According to need? Humanitarian responses to violence in Central America. Humanitarian Exchange Magazine. 2017;69:15-17. Disponível em: https://odihpn.org/magazine/accordingneed-humanitarian-responses-violencecentral-america/ Acessado em agosto de 2018.

6. Escobar-Díaz F, Osorio-Merchán MB, De la Hoz-Restrepo F. Motivos de no vacunación en menores de cinco años en cuatro ciudades colombianas. Rev Panam Salud Publica. 2017;41:e123.

7. Polaro SHI, Gonçalves LHT, Alvarez AM. Enfermeiras desafiando a violência no âmbito de atuação da Estratégia de Saúde da Família. Texto Contexto Enferm. 2013; 22(4):935-42.

8. Machado CB, Daher DV. Violência urbana: repercussões e consequências na assistência à saúde em uma unidade de Saúde da Família. Cien Cuid Saude. 2015;14(4): 1445-52.

9. Organization of American States. Report on the experts meeting: "Searching for common approaches to deal with unconventional conflicts and violence in the Americas". Washington, DC; 2015. Disponível em: https:/ / www.idrc.ca/sites/default/files/ sp/Documents \%20EN/OAS-reportexperts-meeting-unconventional-conflictsAmericas.pdf Acessado em agosto de 2018.

10. Organização Mundial da Saúde (OMS). Declaração de Alma-Ata. Conferência Internacional sobre Cuidados Primários em Saúde. Alma-Ata; 1987. Disponível em: www.who.int/publications/almaata declaration_en.pdf?ua $=1 \& u a=1$ Acessado em agosto de 2018.

11. Starfield B. Atenção primária: equilíbrio entre necessidades de saúde, serviços e tecnologia. Brasília: UNESCO, Ministério da Saúde; 2002. Disponível em: http://bvsms.saude.gov.br/bvs/publicacoes/atencao_primaria_p1.pdf Acessado em agosto de 2018.

12. United Nations. The Sustainable Development Goals Report. Nova Iorque; UN; 2017. Disponível em: https://unstats. un.org/sdgs/report/2018/overview / Acessado em agosto de 2018. relevância da discussão e disparar novas e necessárias reflexões.

Conflitos de interesse. Nada declarado pela autora.

Declaração. As opiniões expressas no manuscrito são de responsabilidade exclusiva da autora e não refletem necessariamente a opinião do Comitê Internacional da Cruz Vermelha nem a opinião ou política da RPSP/PAJPH ou da Organização Pan-Americana da Saúde (OPAS).
13. Butchart A, Mikton C. Global status report on violence prevention, 2014. Technical Report. Genebra: World Health Organization; 2014 Disponível em: http:/ / eprints. uwe.ac.uk/30034 Acessado em agosto de 2018.

14. Starfield, B. Primary care and equity in health: the importance to effectiveness and equity of responsiveness to people's needs. Humanity Soc. 2009;33(1-2):56-73.

15. Gofin J, Gofin R. Atención primaria orientada a la comunidad: un modelo de salud pública en la atención primaria. Rev Panam Salud Publica. 2007;21(2/3):177-85.

16. Minayo MCS. Violência social sob a perspectiva da Saúde Pública. Cad Saude Publica. 1994;10(1):7-18.

17. Favoreto CAO. A prática clínica e o desenvolvimento do cuidado integral à saúde no contexto da atenção primária. Rev APS. 2008;11(1):100-8.

18. Mehry EE, Malta DC, Santos FP. Desafios para os gestores do SUS, hoje; compreender os modelos de atenção à saúde no âmbito da reforma sanitária brasileira e a potência transformadora da gestão. Em: Freese E, editor. Municípios: a gestão da mudança em saúde. Recife: UFPE; 2004. Pp. 45-76.

Manuscrito recebido em 14 de janeiro de 2018. Aceito em versão revisada em 2 de agosto de 2018. 
ABSTRACT The present article aims at discussing the singularities of primary health care (PHC) in contexts where violence, strongly supported by guns, is routinely, chronically, and systemically present, and to gauge the capacity of PHC as empowerment channel for

\section{Primary health care and Latin-American territories marked by violence} communities where armed violence is an expressive vulnerability. Based on the Declaration of Alma-Ata and on the PHC principles described by Barbara Starfield, and focusing on community participation and social determinants of health, the discussion departs from the realities observed in Latin America and the commitment to access and universal coverage set forth in the third Sustainable Development Goal. The qualitative and quantitative characterization of armed violence and its consequences must be qualified using an intersectoral approach with heavy community participation in search of coherent and significant responses. The protection and safety of health care workers must be ensured to guarantee the continuous provision of health care services in contexts of violence.

Keywords Primary health care; violence; social participation; health services accessibility.

RESUMEN En el artículo se trata de abordar los aspectos singulares del trabajo de atención primaria de salud (APS) en los entornos donde la violencia, con el sólido apoyo del uso de armas de fuego, tiene una expresión habitual, crónica y generalizada, y de determinar la posibilidad que ofrece la APS de servir de canal de empoderamiento de la

\section{La atención primaria de} salud y los territorios latinoamericanos marcados por la violencia comunidad, donde la violencia armada representa una vulnerabilidad manifiesta. Con base en la Declaración de Alma-Ata y en los principios de la APS descritos por Bárbara Starfield, y con un enfoque en la participación comunitaria y en la perspectiva de los determinantes sociales de la salud, el debate tiene como punto de partida las realidades observadas en los países de América Latina y los compromisos orientados hacia el acceso universal a la salud y la cobertura universal de salud previstos en el tercero de los Objetivos de Desarrollo Sostenible. La caracterización cualitativa y cuantitativa de la violencia armada y de sus consecuencias debe hacerse a partir de un enfoque intersectorial con amplia participación comunitaria en la búsqueda de respuestas coherentes y significativas. También es preciso garantizar la protección y la seguridad de los profesionales para poder prestar servicios de salud de una manera continua en estos entornos.

Palabras clave
Atención primaria de salud; violencia; participación social; accesibilidad a los servicios de salud. 